\title{
Ginsenoside metabolite 20(S)-protopanaxadiol promotes neural stem cell transition from a state of proliferation to differentiation by inducing autophagy and cell cycle arrest
}

\author{
SHALI CHEN $^{1}$, JI HE ${ }^{2}$, XIYUAN QIN $^{2}$, TIAO LUO $^{3}$, APARNA PANDEY $^{2}$, JIJIA LI $^{3}$, \\ SUYOU LIU ${ }^{2}$, JUNLI LUO ${ }^{2}$, QI WANG ${ }^{4}$ and ZHIYONG LUO ${ }^{2}$
}

\author{
${ }^{1}$ Department of Laboratory Medicine, Heping Hospital Affiliated to Changzhi Medical College, Changzhi, Shanxi 046000; \\ ${ }^{2}$ Department of Biochemistry and Molecular Biology, School of Life Sciences; ${ }^{3}$ Xiangya School of Stomatology, \\ Central South University, Changsha, Hunan 410078; ${ }^{4}$ Department of Pathogen Biology and Immunology, \\ Guangdong Pharmaceutical University, Guangzhou, Guangdong 510008, P.R. China
}

Received June 14, 2019; Accepted February 26, 2020

DOI: $10.3892 / \mathrm{mmr} .2020 .11081$

\begin{abstract}
S)-Protopanaxadiol (PPD) is an active ginseng metabolite and is the final form of protopanaxadiol saponins metabolized by human intestinal microflora. The neuroprotective effects and mechanisms underlying PPD on neural stem cells (NSCs) are not completely understood. The aim of the present study was to assess the effects of PPD on the proliferation and differentiation of neural stem cells. In the present study, following treatment with different concentrations of PPD for $24 \mathrm{~h}$, the percentage of BrdU-positive cells decreased significantly with increasing concentrations of PPD. Moreover, flow cytometric analysis results indicated that PPD treatment increased the proportion of cells in the $\mathrm{G}_{0} / \mathrm{G}_{1}$ and $\mathrm{G} 2 / \mathrm{M}$ phase and decreased the proportion of cells in the $\mathrm{S}$ phase. The activation of autophagy, determined by an increased number of autophagic vacuoles and light chain 3 lipidation, was associated with an increase in the expression of the neuronal marker tubulin- $\beta 3$ following PPD treatment. PPD also partially rescued NSCs from the inhibitory effects of the autophagic inhibitor
\end{abstract}

Correspondence to: Professor Zhiyong Luo, Department of Biochemistry and Molecular Biology, School of Life Sciences, Central South University, 110 Xiangya Road, Changsha, Hunan 410078, P.R. China

E-mail: luozhiyoung@csu.edu.cn

Professor Qi Wang, Department of Pathogen Biology and Immunology, Guangdong Pharmaceutical University, 280 East Outer Ring Road of Guangzhou Mega Center, Guangzhou, Guangdong 510008, P.R. China E-mail: 2449281020@qq.com

Abbreviations: LC3, light chain 3; NSCs, neural stem cells; PPD, 20(S)-protopanaxadiol; PI, propidium iodide; TEM, transmission electron microscopy

Key words: protopanaxadiol, ginsenoside, neural stem cell, differentiation, autophagy wortmannin, suggesting that the effect of PPD on NSC differentiation was associated with autophagy. Collectively, the results indicated that PPD promoted the transition of NSCs from a state of proliferation to differentiation through the induction of autophagy and cell cycle arrest. Therefore, the present study may provide a basis for the development of regenerative therapies based on ginsenoside, an approved and safe drug.

\section{Introduction}

Neurodegenerative diseases, including Parkinson's disease, Alzheimer's disease and amyotrophic lateral sclerosis, are a major cause of disability and death in elderly individuals, which seriously impact the physical and mental health of the affected individual (1-4). With an increasing aged population and the pressure of life, neurological diseases pose a large burden on an individual's life and health; therefore, identifying drugs that protect against neurodegeneration is important.

Ginsenosides are the active ingredients of ginseng, which has been used as a tonic drug in East Asia for $>2,000$ years (5). In recent years, a number of studies have reported that ginsenosides have certain roles in the prevention and treatment of neurological diseases. For example, Ginsenoside Rb1 promoted the proliferation and differentiation of neural stem cells (NSCs) in a rat model of Alzheimer's disease (6). Gerbil models of global ischemia have also indicated the significance of the ginsenosides in neurodegenerative diseases (7). However, research is still in its infancy, and effective physiological dosages and the mechanisms underlying the action of ginsenosides have not yet been fully elucidated. Ginsenosides can be classified into two categories, which display distinct pharmacological roles, based on the functional groups attached at the C6 position: 20(S)-protopanaxadiol (PPD) type and 20(S)-protopanaxatriol type (PPT) (8). In the present study, the effect of the ginsenoside de-glycosylated metabolite PPD on neurogenesis in rat NSC cultures was investigated. The results suggested that the treatment of NSCs with PPD significantly promoted the differentiation of NSCs by inducing autophagy and cell cycle arrest. 


\section{Materials and methods}

NSC cultures. NSCs were prepared as previously described (9). A total of 160 pups were used in the present study. Postnatal day 1 Sprague-Dawley rats (specific pathogen-free grade) were purchased from the Slack Jinda Laboratory Animal Company (Hunan, China). The animals were maintained at $19-22^{\circ} \mathrm{C}$ with $40-50 \%$ humidity, 12-h light/dark cycles. Primary NSCs derived from postnatal day 1 rats were isolated and cultured as follows. Following sacrifice by decapitation, rat brains were dissected in ice-cold DMEM/F12 medium (1:1; Thermo Fisher Scientific, Inc.) supplemented with $2 \%$ penicillin and $50 \mu \mathrm{g} / \mathrm{ml}$ gentamycin. The subventricular zone was treated with $1 \mathrm{ml}$ Stem Pro Accutase (Thermo Fisher Scientific, Inc.) for $10 \mathrm{~min}$ at $37^{\circ} \mathrm{C}$. Following digestion, the samples were filtered using a 200 -mesh sieve and centrifuged at $240 \mathrm{x}$ g for $5 \mathrm{~min}$ at room temperature. The obtained NSCs $\left(5-7 \times 10^{6}\right)$ were cultured in fresh DMEM/F12 supplemented with basic fibroblast growth factor (20 ng/ml; PeproTech, Inc.), epidermal growth factor $(20 \mathrm{ng} / \mathrm{ml}$; PeproTech, Inc.), 2\% B27 (Thermo Fisher Scientific, Inc.), glutamine ( $2 \mathrm{mM}$; Thermo Fisher Scientific, Inc.), penicillin $(50 \mathrm{U} / \mathrm{ml})$ and gentamycin $(50 \mu \mathrm{g} / \mathrm{ml}$; Thermo Fisher Scientific, Inc.) at $37^{\circ} \mathrm{C}$ with $5 \% \mathrm{CO}_{2}$. The culture medium was replaced every 2 days and cells were subcultured every 5 days. Neurospheres were observed on days 1, 3 and 6 using an inverted microscope (magnification, $\mathrm{x} 400$; Olympus Corporation). All procedures involving animals were approved by the Institutional Animal Care and Use Committee of Central South University (Changsha, China). All efforts were made to minimize animal suffering and reduce the number of animals used.

Cell viability and proliferation assays. Cell viability was assessed using the Cell Counting Kit-8 (CCK-8; Vazyme Biotech Co., Ltd.), according to the manufacturer's protocol. Ginsenoside PPD (purity, >95\%) was obtained from the Department of Chemistry, Jilin University, and dissolved in DMSO to make a $100-\mathrm{mg} / \mathrm{l}$ stock solution. Neurospheres (passage 2) were digested to form single-cell suspensions. Cell suspensions $\left(1 \times 10^{5} /\right.$ well) were seeded into 96 -well plates in DMEM/F12 medium containing different concentrations of PPD $(5,10,20$ or $40 \mu \mathrm{M})$. The control group was incubated with DMEM/F12 medium containing 0.1\% DMSO. Following incubation for $72 \mathrm{~h}$ at $37^{\circ} \mathrm{C}, 20 \mu \mathrm{l} \mathrm{CCK}-8$ reagent was added to each well and incubated for a further $4 \mathrm{~h}$ at $37^{\circ} \mathrm{C}$. Absorbance was measured at $450 \mathrm{~nm}$ using a microplate reader (BioTek Instruments, Inc.). The $\mathrm{IC}_{50}$ was calculated according to the optical density values using SPSS software (version 18.0; IBM Corp.).

Cell proliferation was assessed by BrdU incorporation. The single-cell suspensions $\left(1 \times 10^{5}\right.$ cells/well) were plated on a 20 -mm coverslip (Fisherbrand; Thermo Fisher Scientific, Inc.) in a 6-well plates in DMEM/F12 medium containing different concentrations of PPD $(5,10,20$ or $40 \mu \mathrm{M})$. Cells co-cultured with DMEM/F12 medium containing 0.1\% DMSO were used as the control group. All cells were incubated with BrdU (15 $\mu \mathrm{m} / \mathrm{l}$; Sigma-Aldrich; Merck KGaA) for $24 \mathrm{~h}$ at $37^{\circ} \mathrm{C}$ with $5 \% \mathrm{CO}_{2}$ subsequently assessed using an immunofluorescence assay.
Cell cycle assay. The cells were analyzed by flow cytometry (FC 500; Beckman Coulter, Inc.) and analyzing software (CXP 2.1; Beckman Coulter, Inc.). Cells were seeded $\left(1 \times 10^{6}\right.$ cells/well) into 6 -well plates and incubated without or with PPD (10, 20 and $40 \mu \mathrm{M})$ for $24 \mathrm{~h}$ at $37^{\circ} \mathrm{C}$ with $5 \%$ $\mathrm{CO}_{2}$. Cells were harvested and permeabilized overnight at $4^{\circ} \mathrm{C}$ with pre-cooled $75 \%$ ethanol. Subsequently, cells were treated with $1 \mathrm{mg} / \mathrm{ml} \mathrm{RNase}$ A for $30 \mathrm{~min}$ at $37^{\circ} \mathrm{C}$ and stained with $50 \mu \mathrm{g} / \mathrm{ml}$ propidium iodide in the dark for $15 \mathrm{~min}$ at room temperature. The cell cycle was analyzed by flow cytometry.

Cell differentiation assay. Single cell suspensions were seeded $\left(3.5 \times 10^{5}\right.$ cells/well) onto $20-\mathrm{mm}$ round coverslip in 6 -well plates. Cells were cultured in differentiation medium (DMEM/F12 medium without growth factors, but supplemented with 5\% FBS (Gibco; Thermo Fisher Scientific, Inc.) and incubated without or with PPD (10, 20, 40 and $60 \mu \mathrm{M})$ for $72 \mathrm{~h}$ at $37^{\circ} \mathrm{C}$ with $5 \% \mathrm{CO}_{2}$. Differentiation was measured by immunostaining and western blotting for neuronal markers tubulin- $\beta 3$.

Detection of autophagy. Single cell suspensions $\left(1 \times 10^{6}\right)$ were transferred into a culture bottle and cultured in differentiation medium alone or with $20 \mu \mathrm{M}$ PPD for 24,48 or $72 \mathrm{~h}$ at $37^{\circ} \mathrm{C}$ with $5 \% \mathrm{CO}_{2}$. Changes in the expression of autophagy markers, including LC3 and p62 were assessed by western blot analysis. Following cells cultured for 72 h, LC3 punctae formation and autophagic vacuoles were also monitored by immunofluorescence and Transmission electron microscopy (TEM).

To further assess the role of autophagy during NSCs differentiation, NSCs were treated with the autophagic inhibitor wortmannin (WM, $30 \mu \mathrm{M}$, Abcam; cat. no. ab120148) alone or incubated with $20 \mu \mathrm{M}$ PPD in the differentiation medium for $72 \mathrm{~h}$ at $37^{\circ} \mathrm{C}$ with $5 \% \mathrm{CO}_{2}$. Then, neuronal marker Microtubule-Associated Protein 2 (MAP2) expression was assessed by immunofluorescence.

Immunofluorescence. Following fixation with $4 \%$ formaldehyde, pre-coated chamber slides containing the growing cells were washed once with PBS, permeabilized with $0.1 \%$ Triton-100 for $5 \mathrm{~min}$ and blocked with $0.5 \%$ BSA for $30 \mathrm{~min}$. Subsequently, cells were incubated with the following primary antibodies overnight at $4^{\circ} \mathrm{C}$ : Mouse monoclonal anti-BrdU (1:100; Sigma-Aldrich; Merck KGaA; cat. no. NA61), Rabbit monoclonal anti-tubulin- $\beta 3$ (1:100; Abcam; cat. no. ab18207) and Rabbit polyclonal anti-LC3 (1:100; Bioworld Technology, Inc.; cat. no. AP0762). Cells were washed three times with PBS and incubated for $1 \mathrm{~h}$ at $25^{\circ} \mathrm{C}$ with the goat anti-rabbit IgG or goat anti-mouse $\operatorname{IgG}$ (1:300; Abcam; cat. nos. ab97050 and ab97022) secondary antibodies. Cells were washed with PBS and incubated with DAPI (1:200) for $10 \mathrm{~min}$. Stained cells and LC3 punctae were observed in five randomly selected fields of view using an Eclipse confocal fluorescence microscope (Nikon Corporation) and analyzed using Image J 1.46r software (National Institutes of Health).

Western blot analysis. Cells were washed twice with PBS and lysed in RIPA buffer $(50 \mathrm{mM}$ Tris- $\mathrm{HCl}, 150 \mathrm{mM} \mathrm{NaCl}$, $1.0 \mathrm{mM} \mathrm{Na} 3 \mathrm{VO} 4,1 \mathrm{mM}$ EDTA, $1 \% \mathrm{NP}-40,0.5 \%$ sodium 
A

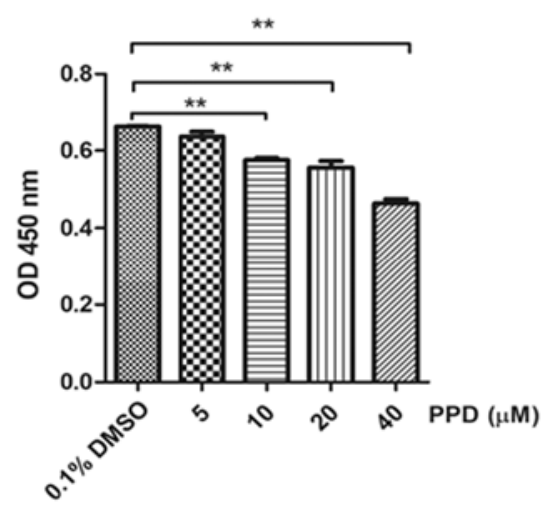

C

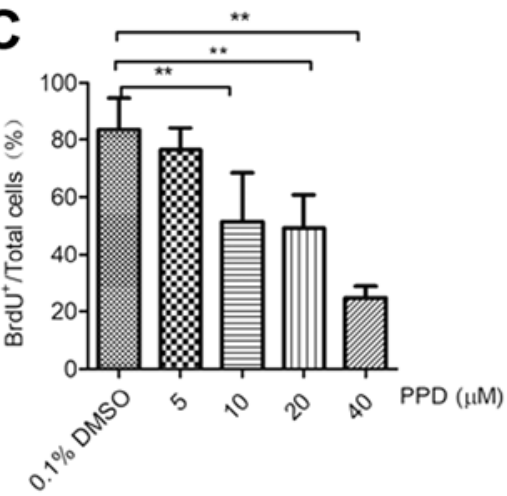

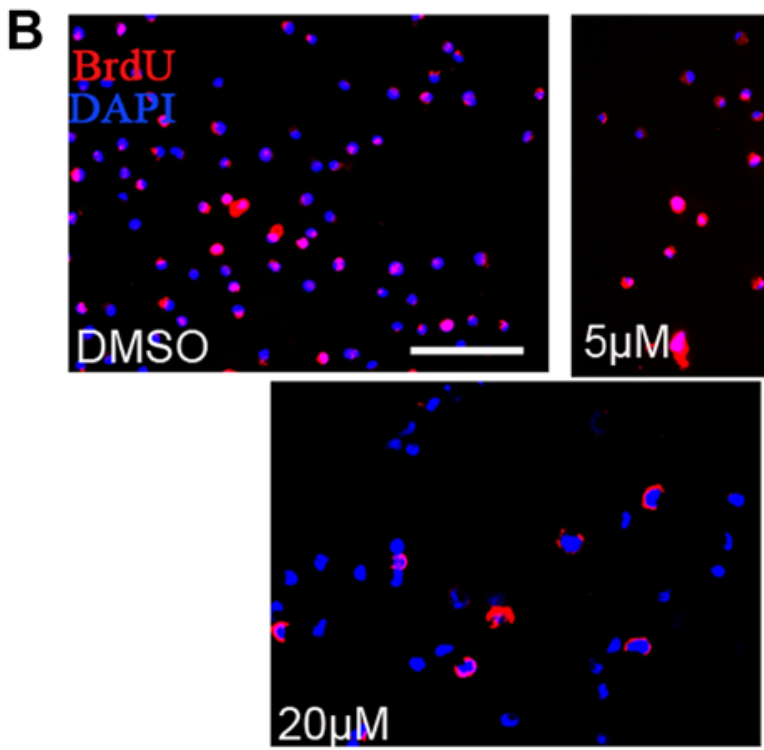
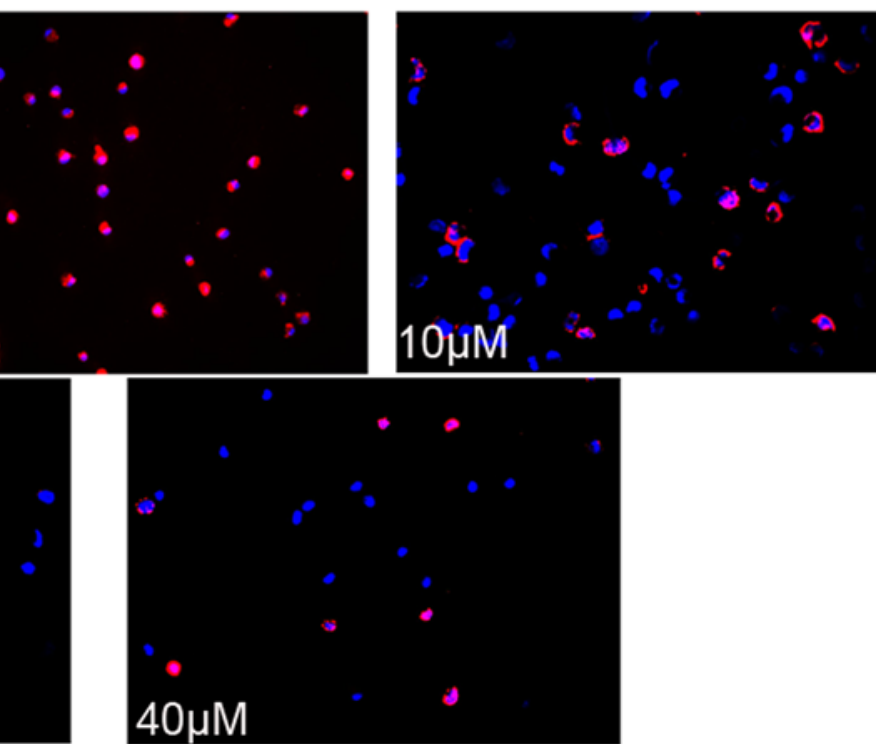

Figure 1. PPD inhibits the viability and proliferation of NSCs in vitro. (A) NSCs were treated with 5, 10, 20 or $40 \mu \mathrm{M}$ PPD for $72 \mathrm{~h}$, and viability was assessed using a Cell Counting Kit-8 assay, cells incubated with DMEM/F12 medium containing 0.1\% DMSO as control group. (B) NSCs were treated with 5, 10, 20 or $40 \mu \mathrm{M}$ PPD for $24 \mathrm{~h}$, and proliferation was analysed using a BrdU incorporation assay, cells incubated with DMEM/F12 medium containing 0.1\% DMSO as control group; scale bar, $50 \mu \mathrm{m}$. (C) Proportion of BrdU-positive cells following PPD treatment. ${ }^{* *} \mathrm{P}<0.01 \mathrm{vs}$. DMSO group. NSCs, neural stem cells; OD, optical density; PPD, 20(S)-protopanaxadiol.

deoxycholate, $0.1 \%$ SDS, $100 \mu \mathrm{g} / \mathrm{ml}$ phenylmethylsulfonyl fluoride, $30 \mu \mathrm{l} / \mathrm{ml}$ aprotinin and $4 \mu \mathrm{g} / \mathrm{ml}$ leupeptin, $\mathrm{pH} 7.5)$. Lysates were centrifuged for $10 \mathrm{~min}$ at $10,000 \mathrm{x} \mathrm{g}$ for $10 \mathrm{~min}$ at $4^{\circ}$. The protein concentrations of the collected supernatants were then determined by bicinchoninic acid Protein Assay kit (Abcam; cat. no. ab102536). Proteins (50 $\mu \mathrm{g}$ per lane) were separated via $10-15 \%$ SDS-PAGE for $1.5 \mathrm{~h}$ at $120 \mathrm{~V}$ and transferred to PVDF membranes for $1.5 \mathrm{~h}$ at $40 \mathrm{~V}$. The membranes subsequently blocked with $5 \%$ skimmed milk in PBS plus $0.1 \%$ Tween-20 (PBST) for $60 \mathrm{~min}$ at room temperature. The membranes were incubated overnight at $4^{\circ} \mathrm{C}$ with primary antibodies $(1: 1,000)$ against Rabbit polyclonal anti-LC3 (Bioworld Technolgies, Inc.; cat. no. AP0762)), Rabbit p62 polyclonal antibody (Bioworld Technolgies, Inc.; cat. no. AP6006), Rabbit monoclonal anti-tubulin- $\beta 3$ (Abcam; cat. no. ab18207) and Rabbit polyclonal $\beta$-actin (Abcam; cat. no. AP0714). LC3-I is $\sim 14 \mathrm{kDa}$ and lipidated LC3 (LC3-II) is $\sim 16 \mathrm{kDa}$. Primary antibodies were diluted in $1 \mathrm{X}$ PBST containing 5\% BSA. Following incubation, the membranes were washed with $1 \mathrm{X}$ PBST and incubated for $2 \mathrm{~h}$ at room temperature with a goat anti-rabbit horseradish peroxidase-conjugated IgG secondary antibody
(1:2,500; Abcam; cat. no. ab97051). Bands were developed with ECL reagent (Thermo Fisher Scientific, Inc.; cat. no. 32209). Protein expression was quantified using Image Lab software 4.1 (Bio-Rad Laboratories, Inc.) with $\beta$-actin as the loading control.

TEM. At the end of incubation, cell monolayers were washed with PBS and scraped gently with a plastic cell scraper. The harvested cells were pelleted by centrifugation at $10,000 \mathrm{x} \mathrm{g}$ for $10 \mathrm{~min}$ at $37^{\circ} \mathrm{C}$. Subsequently, cells were fixed with $4 \%$ glutaraldehyde for $3 \mathrm{~h}$ at $4^{\circ} \mathrm{C}$, post-fixed with $1 \%$ perosmic acid for $2 \mathrm{~h}$ at room temperature, dehydrated with acetone and embedded in an epoxy resin. Serial ultrathin sections $(70 \mathrm{~nm})$ were placed on 400-mesh grids, and double-stained with $2 \%$ uranyl acetate and $0.4 \%$ lead citrate for $15 \mathrm{~min}$ each at room temperature. Sections were observed using a JEM1230 TEM (JEOL, Ltd.) at a magnification of x8,000 in eight randomly selected fields of view. The percentage of autophagic vacuoles area in each per field was calculated.

Statistical analysis. Statistical analyses were performed using SPSS software (version 18.0; IBM Corp.). Data are presented 

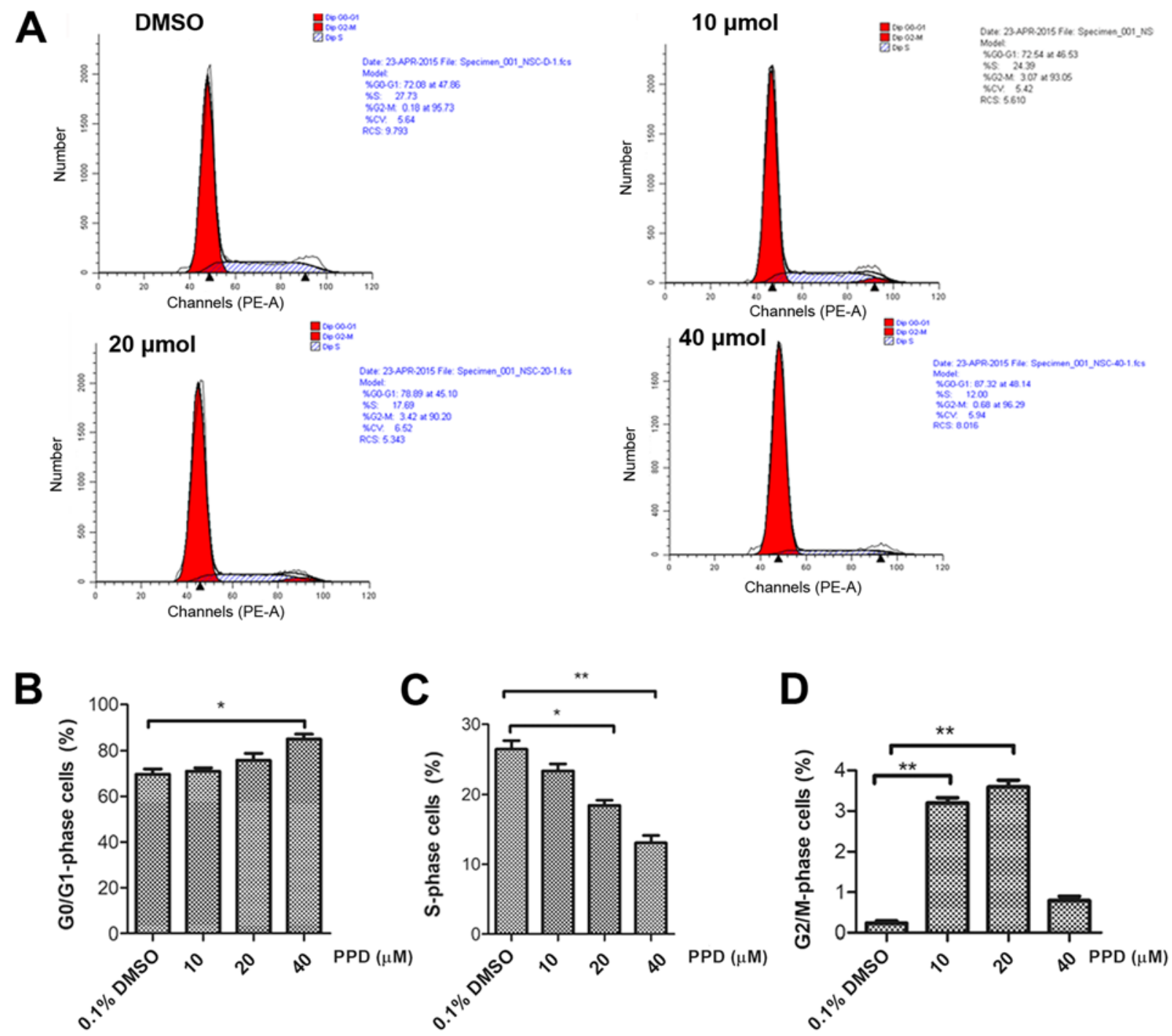

Figure 2. PPD induces cell cycle arrest. (A) Representative plots displaying the distribution of NSCs in the $\mathrm{G}_{0} / \mathrm{G}_{1}, \mathrm{~S}$ and $\mathrm{G}_{2} / \mathrm{M}$ phases following treatment with $0,10,20$ or $40 \mu \mathrm{M}$ PPD for $24 \mathrm{~h}$. The distribution of cells in the (B) $\mathrm{G}_{0} / \mathrm{G}_{1}$, (C) $\mathrm{S}$ and (D) $\mathrm{G}_{2} / \mathrm{M}$ phases. ${ }^{*} \mathrm{P}<0.05$ and ${ }^{* * *} \mathrm{P}<0.01$ vs. DMSO group. NSCs, neural stem cells; PE, phycoerythrin; PPD, 20(S)-protopanaxadiol.

as the mean \pm standard deviation; all analyses were based on biological replicates $(n=3)$ from the same independent experiment. Comparisons between groups were performed using one-way or two-way ANOVA followed by Tukey's post hoc test. $\mathrm{P}<0.05$ was considered to indicate a statistically significant difference.

\section{Results}

PPD inhibits NSC viability and proliferation. To investigate the effect of PPD on NSC proliferation in vitro, NSCs were treated with different concentrations of PPD for $72 \mathrm{~h}$. Cell viability was evaluated using the CCK- 8 assay. Cell viability displayed a dose-dependent relationship with increasing concentrations of PPD (Fig. 1A); the $\mathrm{IC}_{50}$ of PPD was calculated as $180.0 \pm 10.2 \mu \mathrm{M}$. The effect of PPD on cell proliferation was further evaluated using a BrdU incorporation assay. Following treatment with different concentrations of PPD for $24 \mathrm{~h}$, the percentage of BrdU-positive cells in five randomly selected fields of view was calculated for each group. The percentage of BrdU-positive cells decreased significantly with increasing concentrations of PPD compared with the percentage in the control group (Fig. 1B and C). The results indicated that PPD inhibited NSC proliferation in vitro.

$P P D$ induces cell cycle arrest. To further investigate the effect of PPD on NSC proliferation, PPD-induced alterations to the cell cycle were investigated (Fig. 2A). Flow cytometric analysis indicated that PPD treatment increased the proportion of cells in the $\mathrm{G}_{0} / \mathrm{G}_{1}$ phase in a dose-dependent manner from $69.65 \%$ in the control group to $85.10 \%$ in the $40 \mu \mathrm{M}$ PPD group (Fig. 2B). Furthermore, PPD treatment decreased the proportion of cells in the $\mathrm{S}$ phase from $26.53 \%$ in the control group to $13.07 \%$ in the $40 \mu \mathrm{M}$ PPD group (Fig. 2C). The increased proportion of cells in the $G_{2} / M$ phase in the 10 and $20 \mu \mathrm{M}$ PPD groups was significantly higher compared with the control group (Fig. 2D). The results suggested that PPD inhibited NSC proliferation, which may be associated with cell cycle arrest induction. 

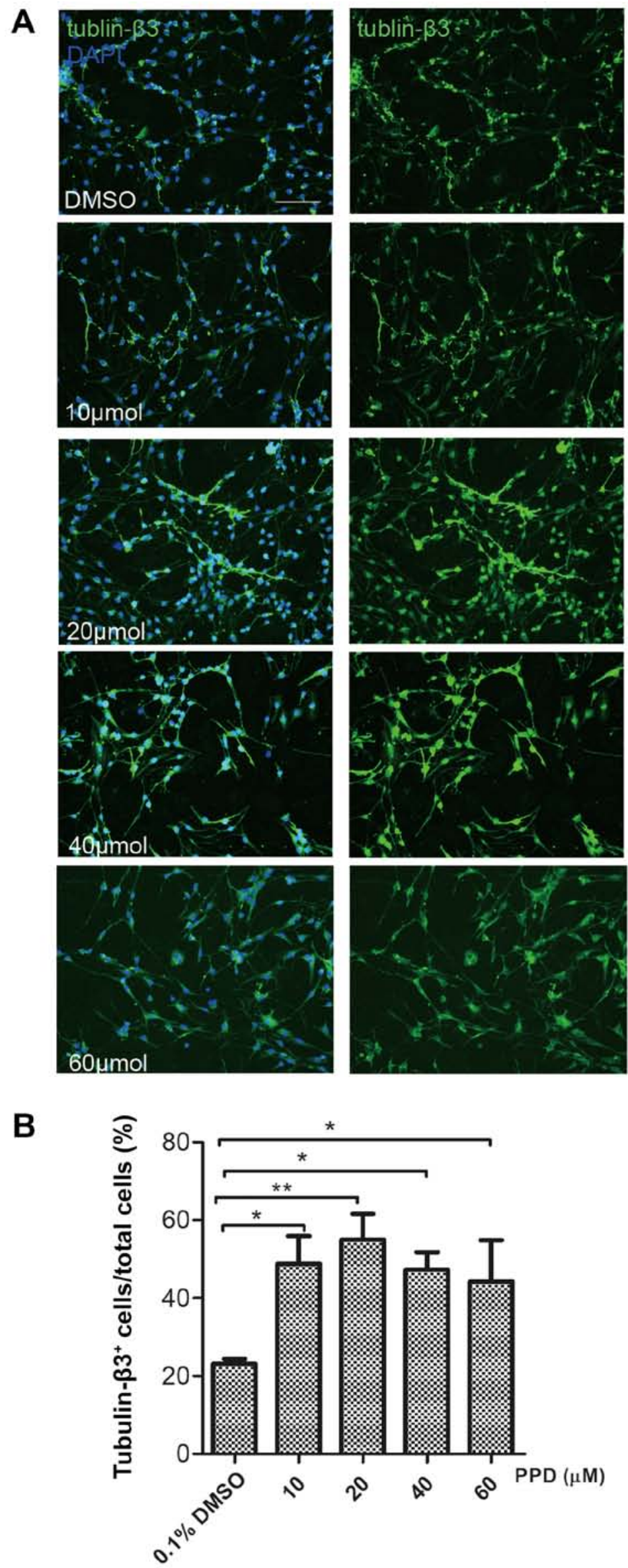

Figure 3. PPD promotes NSC differentiation. NSCs were treated with 10, 20, 40 or $60 \mu \mathrm{M}$ PPD for $72 \mathrm{~h}$. (A) Immunofluorescence images of NSC differentiation following PPD treatment, as indicated by tubulin- $\beta 3$ immunostaining; scale bar, $100 \mu \mathrm{m}$. (B) The proportion of tubulin- $\beta 3$-positive cells. ${ }^{*} \mathrm{P}<0.05$ and ${ }^{* *} \mathrm{P}<0.01$ vs. DMSO group. NSC, neural stem cell; PPD, 20(S)-protopanaxadiol. 

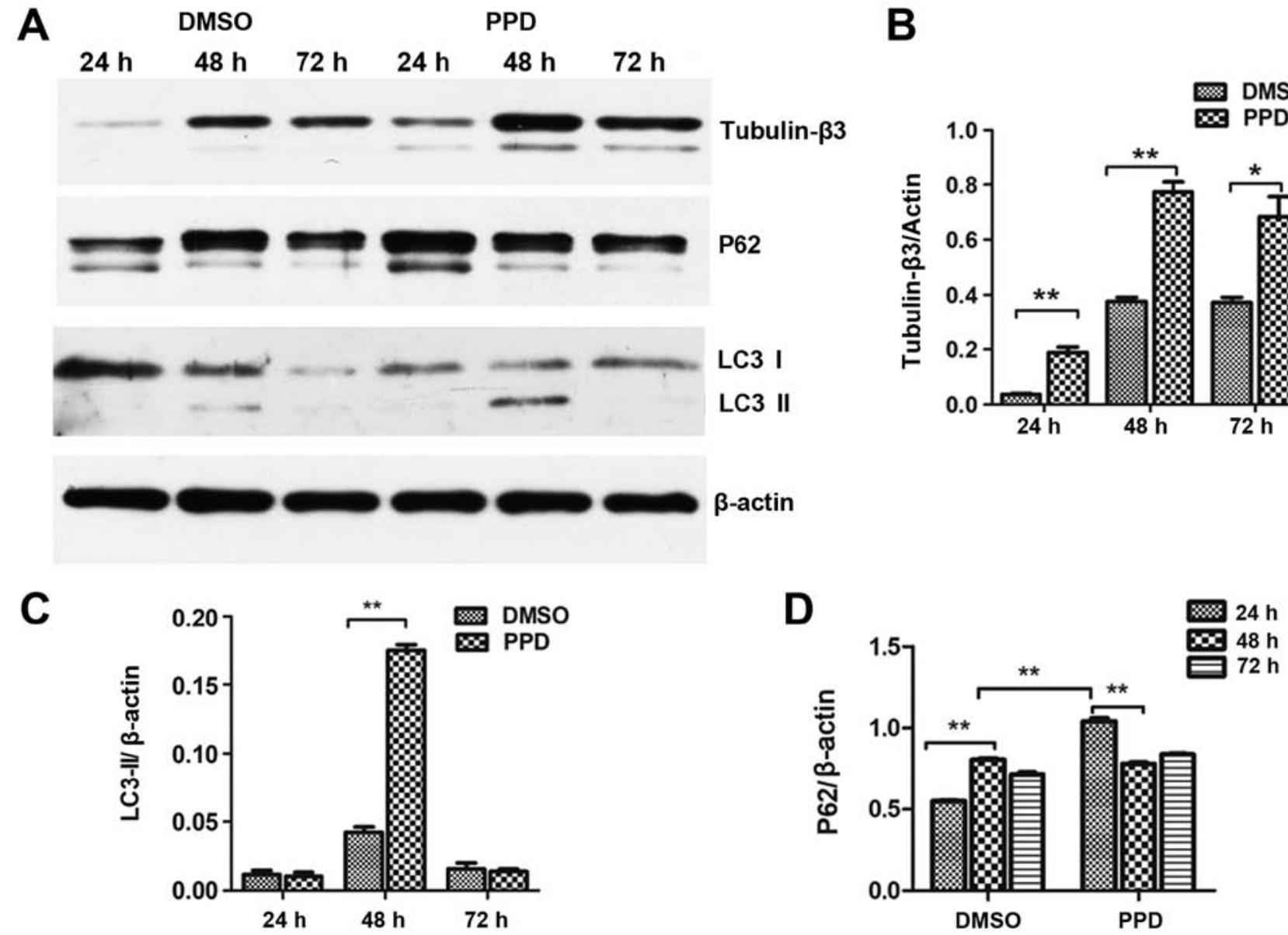

Figure 4. PPD promotes NSC differentiation by inducing autophagy. (A) NSCs were treated with $20 \mu \mathrm{M}$ PPD for 24,48 or $72 \mathrm{~h}$. The indicated protein levels were analyzed by western blotting. (B) Expression of tubulin- $\beta 3$ (neuronal marker) was significantly increased in NSCs following treatment with $20 \mu \mathrm{M}$ PPD. (C) In total, $20 \mu \mathrm{M}$ PPD treatment for $48 \mathrm{~h}$ significantly increased the expression of LC3II compared with the respective control group, (D) p62 expression, which serves as another indicator of autophagy, was increased at $24 \mathrm{~h}$ in the PPD treatment group, which was significantly earlier than that at $48 \mathrm{~h}$ in the control group. ${ }^{*} \mathrm{P}<0.05$ and ${ }^{* *} \mathrm{P}<0.01$ vs. DMSO group. LC3, light chain 3; PPD, 20(S)-protopanaxadiol.

PPD promotes NSC differentiation by inducing autophagy. To investigate the effect of PPD treatment on cell differentiation, immunocytochemistry and western blot assays were performed. After a 72-h culture in differentiation medium, immunofluorescence expression of the neuronal marker tubulin- $\beta 3$ was evaluated. The $20 \mu \mathrm{M}$ PPD group displayed the most significant increase in tubulin- $\beta 3$-positive cells compared with the control group $(\mathrm{P}<0.05$; Fig. $3 \mathrm{~A}$ and $\mathrm{B})$. Western blotting also indicated that the expression level of tubulin- $\beta 3$ was significantly increased in NSCs following treatment with $20 \mu \mathrm{M}$ PPD, compared with the control group at each respective time point $(\mathrm{P}<0.05$; Fig. $4 \mathrm{~A}$ and $\mathrm{B})$. These results indicated that PPD can effectively promote the differentiation of neural stem cells, with the highest effect at $20 \mu \mathrm{m}$. Therefore, in the subsequent experiments, $20 \mu \mathrm{m}$ was selected to study the mechanism of PPD promoting the differentiation of neural stem cells.

To further investigate the effect of PPD on differentiation, NSC autophagy was assessed. LC3 is an essential component of autophagosomes that has been widely used as a marker of autophagy (10). Alterations in the protein expression levels of LC3II were determined by western blotting. It was demonstrated that $20 \mu \mathrm{M}$ PPD treatment for $48 \mathrm{~h}$ significantly increased the expression of LC3II compared with the respective control group $(\mathrm{P}<0.01$; Fig. 4A and $\mathrm{C})$. p62 expression, which often serves as another index of autophagy, increased quickly and significantly at $24 \mathrm{~h}$ after PPD treatment, which was significantly earlier than $48 \mathrm{~h}$ in the control group (Fig. 4A and D). LC3 punctate structures were also detected using an immunofluorescence assay. Treatment with $20 \mu \mathrm{M}$ PPD significantly increased the percentage of LC3 punctae formation compared with the control group $(\mathrm{P}<0.05$; Fig. 5A and B). The TEM results also indicated that the proportion of NSC autophagosomes was higher by PPD treatment compared with the control group (Fig. 5C and D).

To further assess the role of autophagy during NSC differentiation, NSCs were treated with the autophagic inhibitor wortmannin (WM) alone or incubated with $20 \mu \mathrm{M}$ PPD for $72 \mathrm{~h}$ in the differentiation medium. The results suggested that PPD partially rescued NSCs from WM-induced inhibitory effects on MAP2 expression, indicating that the effect of PPD on NSC differentiation was associated with autophagy (Fig. 6A and B).

\section{Discussion}

Generation of new neurons involves proliferation of progenitors, withdrawal from the cell cycle and subsequent differentiation. 

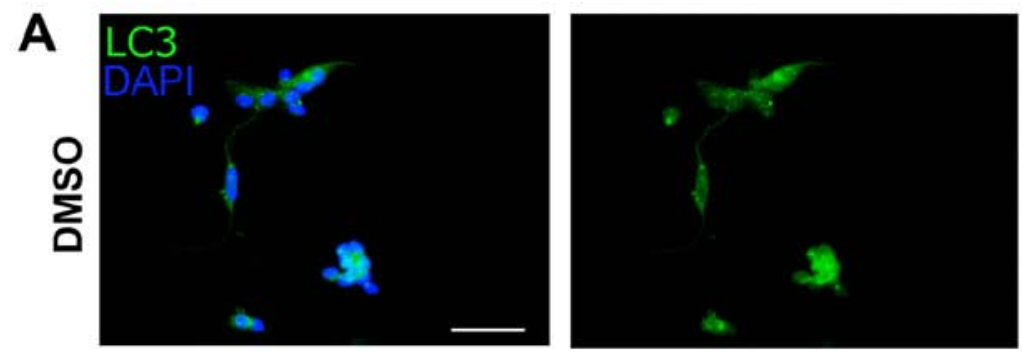

$\mathbf{B}$
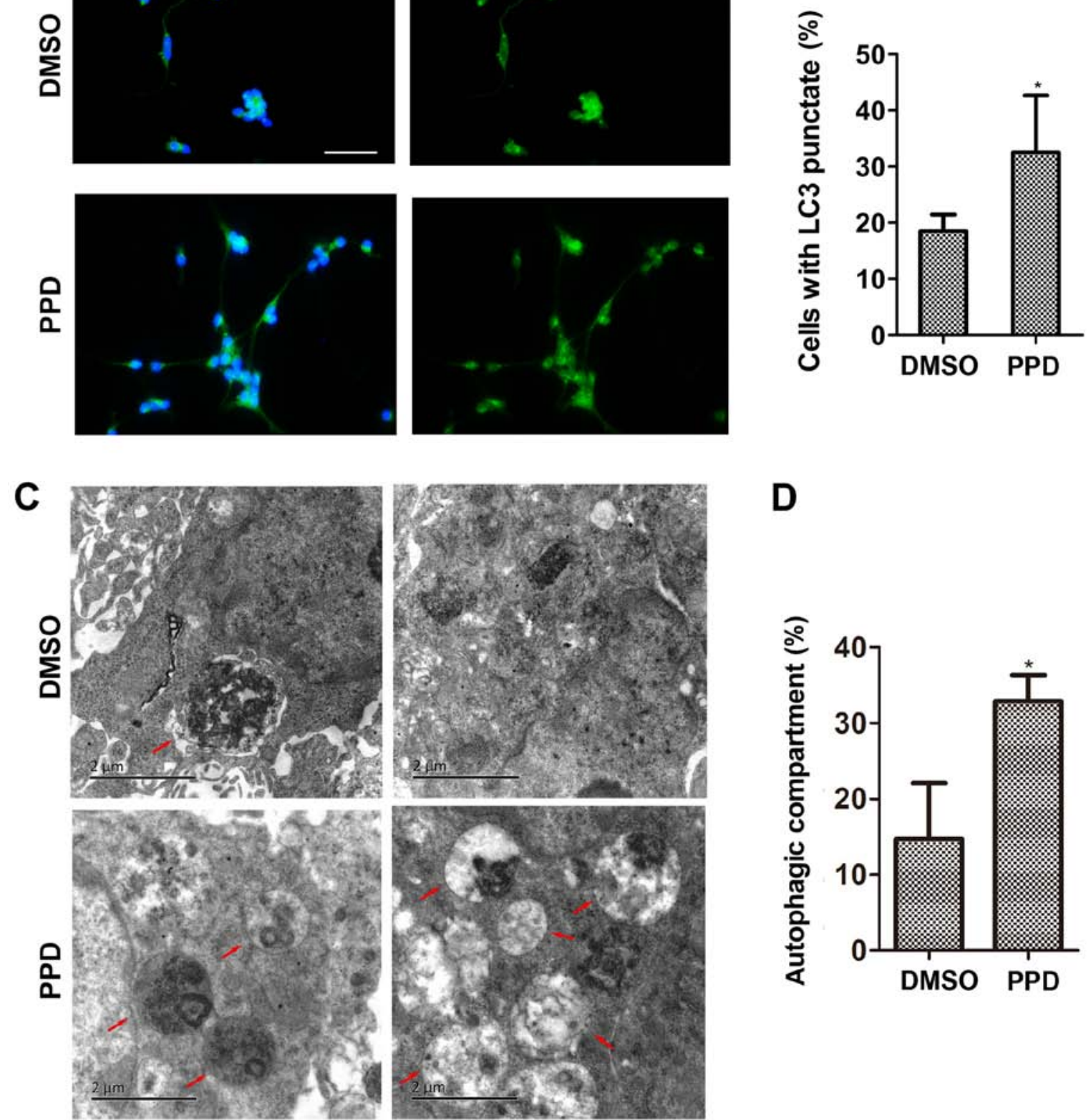

Figure 5. LC3 punctae formation and autophagic vacuoles. (A) Immunofluorescence images of autophagy following treatment with $20 \mu \mathrm{M}$ PPD, as indicated by LC3 immunostaining (scale bar, $100 \mu \mathrm{m}$ ). (B) The percentage of cells with LC3 punctate. (C) Transmission electron microscopy images displayed autophagosome formation in NSCs following treatment with $20 \mu \mathrm{M}$ PPD for $72 \mathrm{~h}$, the arrows indicate autophagic vacuoles. scale bar, $2 \mu \mathrm{m}$. (D) The percentage of autophagic vacuoles area in each per field. ${ }^{*} \mathrm{P}<0.05$ vs. the DMSO group. LC3, light chain 3; PD, 20(S)-protopanaxadiol.

Precursor cells continue to divide before acquiring a fully differentiated state, whereas terminal differentiation usually coincides with proliferation arrest and permanent exit from the cell cycle. The proportion of neural progenitors that remain in and exit from the cell cycle determines the degree of neurogenesis $(11,12)$. The results from the present study suggested that treatment with PPD reduced the proliferation of NSCs, arrested the cell cycle at the $\mathrm{G}_{0} / \mathrm{G}_{1}$ and $\mathrm{G}_{2} / \mathrm{M}$ phases, and promoted NSC differentiation into neurons in vitro. Therefore, it was hypothesized that the therapeutic effect of PPD on nervous system injuries and degenerative disease may occur by promoting NSC cell cycle arrest and triggering neuronal differentiation. The results of the present study were consistent with a previous study, which reported that oral administration of ginsenoside Rb1 significantly increased cell survival but not proliferation in the hippocampus (13). Further investigation into the molecular mechanisms linking NSC cell cycle arrest to cell differentiation is required.

There is evidence for an active role for autophagy during NSC differentiation. Vázquez et al (14) reported an increase in the expression levels of the autophagy genes Autophagy Related 7, Beclin1, activating molecule in beclin1-regulated autophagy (Ambra1) and LC3 in the mouse embryonic olfactory bulb during the initial period of neuronal differentiation, along with a parallel increase in neuronal markers. Furthermore, Fimia et al (15) revealed that Ambral knockout in mouse embryos leads to severe neural tube defects associated 

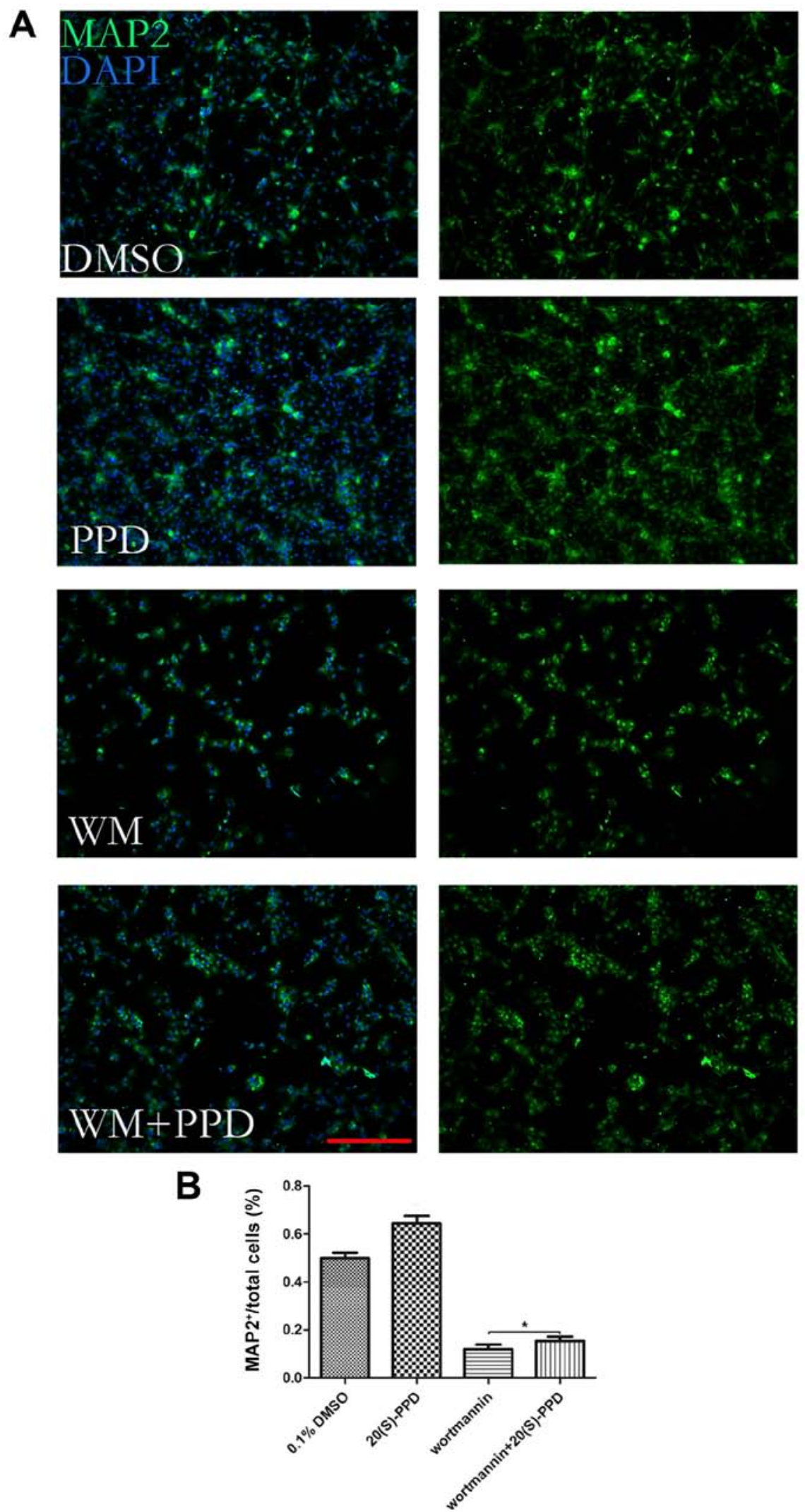

Figure 6. PPD partially rescues NSCs from WM-induced inhibitory effects on MAP2 expression. (A) Following treatment with or without PPD and WM, cells were immunostained for the neuronal marker MAP2; scale bar, $200 \mu \mathrm{m}$. (B) The proportion of MAP2-positive cells. " $\mathrm{P}<0.05$ vs. the DMSO group. MAP2, microtubule-associated protein 2; PPD, 20(S)-protopanaxadiol; WM, wortmannin.

with autophagy impairment, the accumulation of ubiquitinated proteins, unbalanced cell proliferation and excessive cell death. Chemical inhibitors, including 3-methyladenine and LY294002, can reverse retinoic acid-induced neuronal differentiation of neuroblastoma N2a cells, and RNA interference of Beclin 1 significantly delays this process (16). 
Results from the present study indicated that LC3II expression was significantly increased following treatment with PPD for $48 \mathrm{~h}$ compared with the control group. The p62 expression, which often serves as another index of autophagy, increased quickly and significantly at $24 \mathrm{~h}$ after PPD treatment, which was significantly earlier than $48 \mathrm{~h}$ in the control group. Previous studies have reported that p62 protein, via LC3, might be involved in facilitating the clearance of polyubiquitinated protein aggregates by linking the aggregates to the autophagic machinery $(17,18)$. Deterioration of the p62 promoter results in a blockade of p62 expression and can also impair the autophagic elimination of Tau aggregates (18). Based on the results of the present study, it was hypothesized that PPD may accelerate the process of linking polyubiquitinated protein aggregates to the autophagic machinery, which may also be the mechanism of PPD inhibiting NSCs proliferation and promoting cell differentiation. Future studies investigating the mechanisms underlying the effects of PPD on NSC differentiation and survival are required to verify the results of the present study.

In conclusion, the results indicated that PPD inhibited NSC proliferation and promoted NSC differentiation, potentially through a mechanism associated with autophagy and cell cycle arrest. However, the present study was only preliminary and included a number of limitations, such as the lack of in vivo experiments and a failure to present data regarding alterations to the expression levels of LC3II and tubulin- $\beta 3$ in the presence of the autophagy inhibitor WM. The present study may provide a theoretical basis for the development of novel regenerative therapeutic strategies using ginsenoside, an approved and safe drug.

\section{Acknowledgements}

Not applicable.

\section{Funding}

The present study was supported by The National Natural Science Foundation of China (grant nos. 81673544, 81973710 and 81903107), The Hunan Provincial Natural Science Foundation of China (grant nos. 2016JJ4113 and 2018SK2110), The Hunan Innovation Projects for University Students in 2016, Xiangya Hospital Central South University Natural Science Foundation for the Youth (grant no. 2014Q06), and The Changzhi Medical College Research Startup Fund (grant no. QDZ201523).

\section{Availability of data and materials}

All data generated or analyzed during this study are included in this published article.

\section{Authors' contributions}

ZL, QW and JL conceived and designed the study. SC, JH, XQ, TL, SL and AP performed the experiments and data analyses. ZL, QW, SC and AP drafted the manuscript and figures.

\section{Ethics approval and consent to participate}

The present study was approved by the Institutional Animal Care and Use Committee of Central South University.

\section{Patient consent for publication}

Not applicable.

\section{Competing interests}

The authors declare that they have no competing interests.

\section{References}

1. Blin P, Dureau-Pournin C, Foubert-Samier A, Grolleau A Corbillon E, Jové J, Lassalle R, Robinson P, Poutignat N, Droz-Perroteau C and Moore N: Parkinson's disease incidence and prevalence assessment in France using the national healthcare insurance database. Eur J Neurol 22: 464-471, 2015.

2. Bhullar KS and Rupasinghe HP: Polyphenols: Multipotent therapeutic agents in neurodegenerative diseases. Oxid Med Cell Longev 2013: 891748, 2013.

3. Rocca WA, Petersen RC, Knopman DS, Hebert LE, Evans DA Hall KS, Gao S, Unverzagt FW, Langa KM, Larson EB and White LR: Trends in the incidence and prevalence of Alzheimer's disease, dementia, and cognitive impairment in the United States. Alzheimers Dement 7: 80-93, 2011.

4. Davinelli S, Maes M, Corbi G, Zarrelli A, Willcox DC and Scapagnini G: Dietary phytochemicals and neuro-inflammaging: From mechanistic insights to translational challenges. Immun Ageing 13: 16, 2016.

5. Shin BK, Kwon SW and Park JH: Chemical diversity of ginseng saponins from Panax ginseng. J Ginseng Res 39: 287-298, 2015.

6. Zhao J,Lu S, Yu H, Duan S and Zhao J: Baicalin and ginsenoside Rb1 promote the proliferation and differentiation of neural stem cells in Alzheimer's disease model rats. Brain Res 1678: 187-194, 2018.

7. Cheng Y, Shen LH and Zhang JT: Anti-amnestic and anti-aging effects of ginsenoside Rg1 and Rb1 and its mechanism of action. Acta Pharmacol Sin 26: 143-149, 2005.

8. Wong $\mathrm{A}$, Che $\mathrm{C}$ and Leung $\mathrm{K}$ : Recent advances in ginseng as cancer therapeutics: A functional and mechanistic overview. Nat Prod Rep 32: 256-272, 2015.

9. Marshall GP II, Ross HH, Suslov O, Zheng T, Steindler DA and Laywell ED: Production of neurospheres from CNS tissue. Methods Mol Biol 438: 135-150, 2008.

10. Klionsky DJ, Abdelmohsen K, Abe A, Abedin MJ, Abeliovich $\mathrm{H}$, Acevedo Arozena A, Adachi H, Adams CM, Adams PD, Adeli K, et al: Guidelines for the use and interpretation of assays for monitoring autophagy ( $3^{\text {rd }}$ edition). Autophagy 12: 1-222, 2016.

11. Ruijtenberg S and van den Heuvel S: Coordinating cell proliferation and differentiation: Antagonism between cell cycle regulators and cell type-specific gene expression. Cell Cycle 15: 196-212, 2016

12. Zhang RL, Zhang ZG, Roberts C, LeTourneau Y, Lu M, Zhang L, Wang Y and Chopp M: Lengthening the G(1) phase of neural progenitor cells is concurrent with an increase of symmetric neuron generating division after stroke. J Cereb Blood Flow Metab 28: 602-611, 2008.

13. Liu L, Hoang-Gia T, Wu H, Lee MR, Gu L, Wang C, Yun BS, Wang Q, Ye S and Sung CK: Ginsenoside Rb1 improves spatial learning and memory by regulation of cell genesis in the hippocampal subregions of rats. Brain Res 1382: 147-154, 2011.

14. Vázquez P, Arroba AI, Cecconi F, de la Rosa EJ, Boya P and de Pablo F: Atg5 and Ambra1 differentially modulate neurogenesis in neural stem cells. Autophagy 8: 187-199, 2012.

15. Fimia GM, Stoykova A, Romagnoli A, Giunta L, Di Bartolomeo S, Nardacci R, Corazzari M, Fuoco C, Ucar A, Schwartz P, et al: Ambral regulates autophagy and development of the nervous system. Nature 447: 1121-1125, 2007.

16. Zeng $\mathrm{M}$ and Zhou JN: Roles of autophagy and mTOR signaling in neuronal differentiation of mouse neuroblastoma cells. Cell Signal 20: 659-665, 2008.

17. Shvets E, Fass E, Scherz-Shouval R and Elazar Z: The N-terminus and Phe52 residue of LC3 recruit p62/SQSTM1 into autophagosomes. J Cell Sci 121: 2685-2695, 2008.

18. Pankiv S, Clausen TH, Lamark T, Brech A, Bruun JA, Outzen H, Øvervatn A, Bjørkøy G and Johansen T: p62/SQSTM1 binds directly to Atg8/LC3 to facilitate degradation of ubiquitinated protein aggregates by autophagy. J Biol Chem 282: 24131-24145, 2007. 